\title{
A dusty torus around the luminous young star $\operatorname{LkH} \alpha 101$
}

\author{
Peter G. Tuthill*, John D. Monnier ${ }^{\dagger} \&$ William C. Danchi ${ }^{\ddagger}$
}

\begin{abstract}
* Astronomy Department, School of Physics, Univ of Sydney, Sydney NSW 2006, Australia.
$\dagger$ Harvard-Smithsonian Center for Astrophysics, 60 Garden St, Cambridge, MA 02138, USA.

$\ddagger$ NASA Goddard Space Flight Center, Infrared Astrophysics, Code 685, Greenbelt, MD 20771, USA.
\end{abstract}

A star forms when a cloud of dust and gas collapses. It is generally believed that this collapse first produces a flattened rotating disk [1], 2], through which matter is fed onto the embryonic star at the center of the disk. When the temperature and density at the center of the star pass a critical threshold, thermonuclear fusion begins. The remaining disk, which can still contain up to $\sim 0.3$ times the mass of the star [3, 4, 5], is then sculpted and eventually dissipated by the radiation and wind from the newborn star. Unfortunately this picture of the structure and evolution of the disk remains speculative because of the lack of morphological data of sufficient resolution and uncertainties regarding the underlying physical processes. Here we present resolved images of a young star, $\mathrm{LkH} \alpha 101$ in which the structure of the inner accretion disk is resolved. We find that the disk is almost face-on, with a central gap (or cavity) and a hot inner edge. The cavity is bigger than previous theoretical predictions [6], and we infer that the position of the inner edge is probably determined by sublimation of dust grains by direct stellar radiation, rather than by disk reprocessing or the viscous heating processes as usually assumed[7].

The Herbig Ae/Be stars, thought to be pre-main-sequence stars of intermediate mass, have generated considerable recent controversy over their circumstellar structure. Accretion-disk models were found to fit the spectral energy distribution (SED) provided that an optically thin hole or cavity was allowed around the central star [6, 8]. This model drew almost immediate criticism over missing accretion luminosity [9] and problems with forbidden emission line profiles not matching expectations[10]. Further confusion arose from the finding that the SED could be fitted by spherically-symmetric circumstellar shells [11, 12] or composite shell-disk models 13.

$\mathrm{LkH} \alpha 101$ is amongst the brightest young stellar objects in the near-infrared, despite its location behind considerable line-of-sight obscuration (recent estimates of visible extinction $A_{v}$ range from 9.4[14] to 18.5[15]). Interferometric observations with the Keck I telescope capable of imaging structure on scales of tens of milli-arcseconds[16] have enabled us to observe the circumstellar environment of $\mathrm{LkH} \alpha 101$ in the near-infrared at an unprecedented level of detail. 
Images presented in Figure 1, taken in the $\mathrm{H}$ and $\mathrm{K}$ bands, show the bright core to be resolved into a circular limb-brightened disk with a marked asymmetric brightening along the southwest side. A region of suppressed flux, or dark hole, is apparent at the center. $\mathrm{LkH} \alpha 101$ is also revealed as a binary star, with a companion at a separation of 180 mas to the E-NE in the $1.65 \mu \mathrm{m}$ map. This binary component is still visible in the map at $2.27 \mu \mathrm{m}$, although it is now much fainter, appearing only at the lowest contour with a signal-to-noise ratio around 1. The relative brightening of the companion from $\mathrm{K}$ to $\mathrm{H}$ band implies a significantly hotter apparent spectrum.

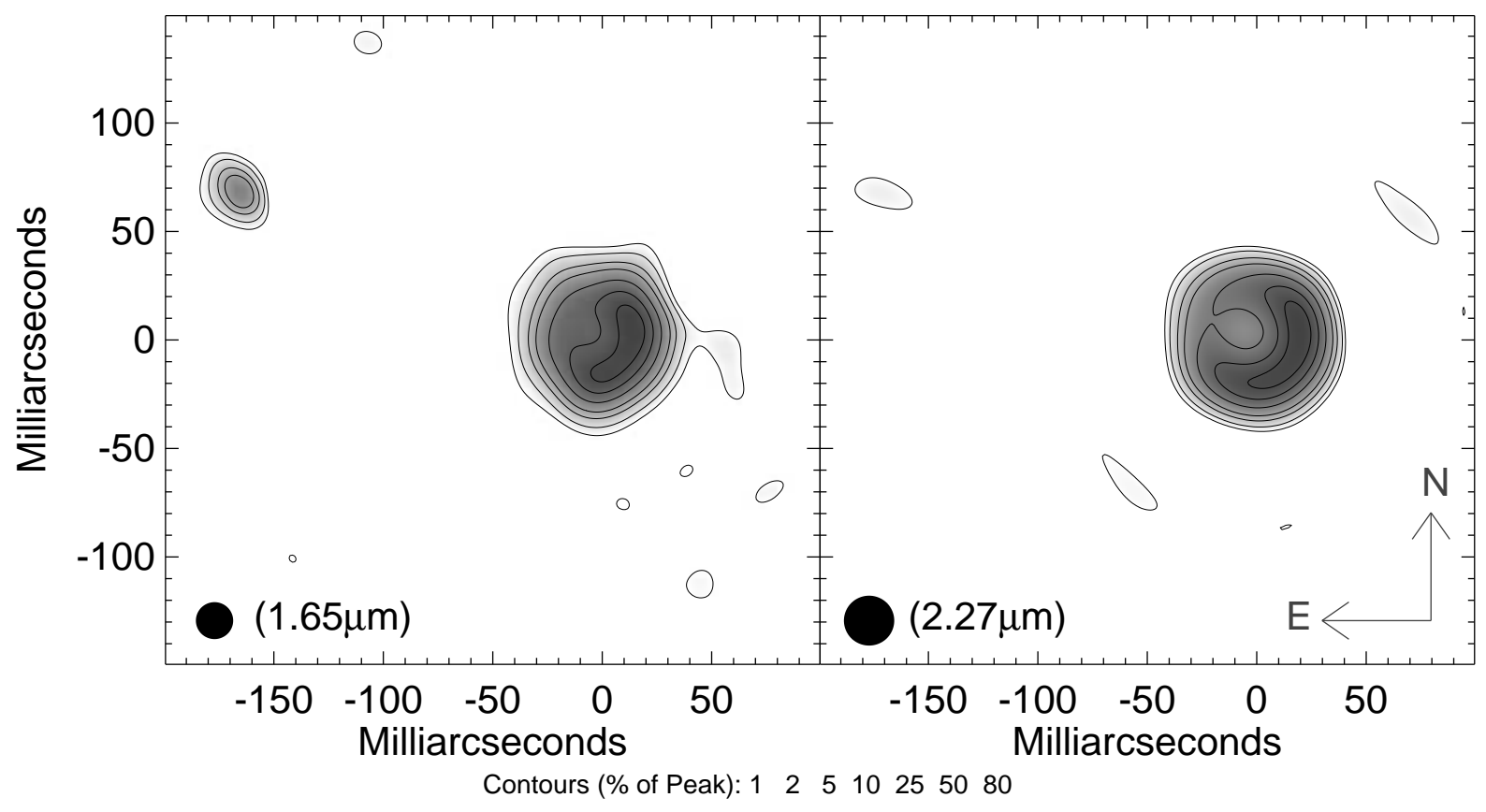

Figure 1: Maps of the $\mathrm{LkH} \alpha 101$ system. Maximum-Entropy image reconstructions at 1.65 (left) and $2.27 \mu \mathrm{m}$ (right) from data taken in September 1998 (JD 2451086). Filter bandwidths were 0.33 and $0.16 \mu \mathrm{m}$ respectively. As it is difficult to extract both absolute astrometry and photometry with our interferometric techniques, the origin has been centered on the disk and images scaled to the brightest pixel. The K-band map is of slightly superior quality to $\mathrm{H}$ band due to the less-detrimental impact of the seeing at longer wavelengths. High spatial resolution information was extracted from high magnification $(20 \mathrm{mas} /$ pixel) fast-readout $(\sim$ $130 \mathrm{~ms})$ data frames taken with an annulus-shaped aperture mask[16]. Consecutive sets of 100 images, interleaved between $\mathrm{LkH} \alpha 101$ and nearby point-source objects, allowed calibration of the statistical behavior of the telescope-atmosphere point-spread function. Data were processed to extract Fourier bispectral data, with the final output being a calibrated set of 630 Fourier amplitudes and 7140 closure phases. High-quality images were produced from an algorithm based on the maximum entropy method[26, 27], although other methods such as CLEAN have also been utilized with near-identical results. The fidelity of our mapping process has been extensively tested on binary stars and other known compact asymmetric targets 28, 29].

The circular appearance of the resolved primary component implies a face-on viewing an- 
gle onto an accretion disk. Fitting to the images in Figure 1 gives a maximum possible inclination angle of about $35^{\circ}$ to the line-of-sight. The central star is not seen at either wavelength, as its contribution is swamped by the extremely high infrared luminosity of the disk. By examining the visibility curves, we find an upper limit of $5 \%$ for any unresolved component in the maps. This should not be surprising as the infrared excesses exhibited by many Herbig Ae/Be stars imply that circumstellar material completely dominates the SED from the infrared through to the radio wavelengths.

The depression or hole in the emission that we saw at the center of the ring provides definitive confirmation of the scenario of an accretion disk with a central optically thin cavity in this young Herbig Ae/Be system. Although this finding is in accord with the scenario of Hillenbrand et al [6], this agreement may be superficial as the cavity observed here is much larger than those considered previously.

The asymmetric flux resulting in the bright crescent appearance to the south-west is a result of the disk being tilted away from the line-of-sight in this direction. This allows us to see through the central cavity to the hot inner edge of the disk on the far side (in the south-west), while in the north-east our view is partially blocked by cooler regions of the disk in the line-of-sight. Just such a "peculiar horseshoe-like feature" was well predicted [8] from models with simulated disks tilted at an angle of $30^{\circ}$, albeit in the mid-infrared $(\lambda=12 \mu \mathrm{m})$ wavelengths.

In order to make meaningful comparison with literature models, we need to estimate the basic properties of the central star driving $\mathrm{LkH} \alpha 101$. The extremely high absolute bolometric luminosity of $4.8 \times 10^{4} L_{\odot}$ [14], where $L_{\odot}$ is the luminosity of the Sun, some $75 \%$ of which is thought be coming from a deeply-embedded cluster containing hundreds of objects 17, was based on an earlier distance determination of $800 \mathrm{pc} 18$. However, a recent distance estimate mainly based on radio-photometry points to $160 \mathrm{pc}[19]$ - typical of the TaurusAuriga star formation complex with which $\mathrm{LkH} \alpha 101$ may be associated. If this distance is correct, the implied intrinsic luminosity (a factor of $\sim 25$ times lower ) is $4.8 \times 10^{2} L_{\odot}-$ more appropriate for a mid-late B star than an O star as was supposed. However, radio observations showing an ionized shell 20, 21] imply Lyman continuum photon fluxes such as would result from a much hotter early B star (B0 to B1). Given the uncertainties in the extinction and the known global anisotropies, an exact classification of $\mathrm{LkH} \alpha 101$ is problematic, but assuming it to be an early B-type zero age main sequence (ZAMS) star gives an approximate mass $M_{\star}$ of $5-10 M_{\odot}$, an effective temperature $T_{\text {eff }} \sim 20000 \mathrm{~K}$ and a radius $R_{\star} \sim 4 R_{\odot}[22]$ ).

The circular ridge of brightest emission has a radius of 21 mas measured from the K-band map of Figure 1. If we identify this as the hot edge of an inwardly-terminated accretion $\operatorname{disk}[8]$, this implies a physical radius of $3.4 \mathrm{AU}$ for a distance of $160 \mathrm{pc}$ (or alternatively $16.8 \mathrm{AU}$ at $800 \mathrm{pc}$ ). This is vastly larger than the $3<R_{\text {hole }} / R_{\star}<25$ inner cavities required by Hillenbrand et al. [6] to fit the near-infrared inflections in the SED. For example, models of early B stars from this sample had derived cavity radii of $0.3-0.6 \mathrm{AU}$, around an order of magnitude smaller than the one we see. Although we find some qualitative agreement, we are forced to conclude that our images are inconsistent with classical accretion disk models. 
This reinforces findings from recently reported high-resolution observations of the Herbig objects with the Infrared Optical Telescope Array (IOTA) 23, 24] and the Palomar Testbed Interferometer 25]. Although most of the visibility data fitted with standard viscously-heated accretion disks $\left(T \propto R^{\frac{-3}{4}}\right)$, an unlikely set of extreme inclination angles was required[24]). Instead, simpler spherical Gaussian or ring models worked better, suggesting that the ensemble of observations were most consistent with spherical shell geometries. The one-sided banana-shaped brightening on our images does not suggest spherical symmetry. The IOTA, with limited sky rotation (typically $\sim 30^{\circ}$ baseline rotation) and Fourier coverage may not have detected similar asymmetries if present in their targets. The match between the asymmetric limb-brightened structure we see and the models of Kessel et al. [B] supports models based on the idea of an accretion disk with a central cavity for LkH $\alpha 101$. Resolved imaging of more systems will be required to settle the conflict between spherical versus disk models for Herbig Ae/Be stars.

There is a rapidly-developing theoretical framework for interpreting young stellar objects at later epochs where the winds and radiation from the fully-luminous central star sculpt the remnant accretion disk by photo-evaporation and wind ram pressure. By equating the plasma sound speed with the Keplerian orbital velocity, Hollenbach et al. [5] defined the gravitational radius $r_{g}$ beyond which material is no longer gravitationally bound and will escape as an ionized wind. Following these authors, we find for a $7.5 M_{\odot}$ star that $r_{g}=50 \mathrm{AU}$, although this does entail extrapolation to less massive stars than were originally considered (alternatively $r_{g}=100 \mathrm{AU}$ if the heavier $15 M_{\odot}$ stellar mass 17 is adopted). This is significantly larger than the apparent structure we see, implying the resolved disk fits well within the picture of a gravitationally bound circumstellar structure.

The photo-evaporative models [8] were constructed for an $8.4 M_{\odot}$ star surrounded by a 1.6 $M_{\odot}$ circumstellar disk - not dissimilar to our expectations for $\mathrm{LkH} \alpha$ 101. Radiative and hydrodynamical modeling resulted in inner disk cavities around $40 \mathrm{AU}$ in radius. Thus the model scale is larger than we observed by almost an order of magnitude, although this discrepancy would vanish if the $800 \mathrm{pc}$ distance scale is adopted. However, the visual match of the appearance of disk models inclined at $30^{\circ}$ [8] and the observed limb-brightened crescent of Figure 1 encourage hope that the basic structure and physics of radiant and wind processes describing the sculpting and illumination of the stellar disk are on the right track. Perhaps self-consistent models can be found with closer inner radii.

We proceed with a far simpler comparison of our 3.4 AU cavity with the radius at which dust sublimates due to heating from the stellar radiation field: $R_{s}=\frac{1}{2} \sqrt{Q}_{R}\left(T_{\star} / T_{s}\right)^{2} R_{\star}$ where $T_{s}$ is the sublimation temperature and $Q_{R}=Q\left(T_{\star}\right) / Q\left(T_{s}\right)$ the ratio of the absorption efficiencies $Q(T)$ of the dust for radiation at color temperature $T$ of the incident and reemitted field. Assuming that grains surviving at the inner edge will be those best able to radiate away the energy from the star (blackbody grains) we assume $Q_{R}=1$. Taking $T_{s}=1500 \mathrm{~K}$ and using the stellar parameters above gives $R_{s}=1.7 \mathrm{AU}-$ a reasonable match to our observed cavity given the uncertainties in the stellar properties. Also, our fit was to the peak of the ridge of emission in Figure 1, but the innermost edge may be somewhat smaller than this. Finding the dust at (or somewhat beyond) the sublimation radius is analogous to similar findings for asymptotic giant branch stars, implying that the same 
basic radiative equilibrium processes may set the inner cavity size in both cases.

We turn now to the binary companion which was found to contribute some $0.8 \%$ of the flux at $\mathrm{K}$ and $4.0 \%$ of the flux at $\mathrm{H}$. Such a luminous (relatively) blue object may be the source of Lyman-continuum photoionizing flux. Alternatively the (unseen) object at the center of the disk may power the energetic emission-line spectrum. Given the flux ratios in the near-infrared, it is possible to estimate the temperature of the companion relative to the disk. Unfortunately, the uncertainties in the extinction mentioned above hamper the de-reddening calculations, so we can only place limits, finding the companion to be at least $2000 \mathrm{~K}$ hotter than the disk.

We detected the secondary at multiple epochs and the components show no apparent relative motion greater than $15 \mathrm{mas} / \mathrm{yr}$. As it seems reasonable to assume that the two objects do form a bound pair, assuming a total system mass of $\sim 10 M_{\odot}$ results in an orbital period of $49 \mathrm{yr}$ given a physical separation of $28.8 \mathrm{AU}$. The apparent motion exhibited by such a system is comparable to our limit, and so extra observations at future epochs should reveal the orbit of this system. We hope such future observations, and the extension of imaging to shorter wavelengths, will help to identify which component provides the ionizing flux, powering this young active region.

\section{References}

[1] Terebey, S., Shu, F.H. \& Cassen, P. The collapse of the cores of slowly rotating isothermal clouds. Astrophys J., 286, 529-551 (1984)

[2] Yorke, H.W., Bodenheimer, P. \& Laughlin, G. The formation of protostellar disks. 2: Disks around intermediate-mass stars. Astrophys J., 443, 199-208 (1995)

[3] Cassen, P.M., Smith, B.F., Miller, R.H. \& Reynolds, R.T. Numerical experiments on the stability of preplanetary disks Icarus, 48, 377-392 (1981)

[4] Shu, F.H., Tremaine, S., Adams, F.C. \& Ruden, S.P. Sling amplification and eccentric gravitational instabilities in gaseous disks. Astrophys J., 358, 495-514 (1990)

[5] Hollenbach, D., Johnstone, D., Lizano, S. \& Shu, F. Photoevaporation of disks around massive stars and application to ultracompact H II regions. Astrophys J., 428, 654-669 (1994)

[6] Hillenbrand, L.A., Strom, S.E., Vrba, F.J. \& Keene, J. Herbig Ae/Be stars Intermediate-mass stars surrounded by massive circumstellar accretion disks. Astrophys J., 397, 613-643 (1992)

[7] Lynden-Bell, D. \& Pringle, J.E. The evolution of viscous discs and the origin of the nebular variables Mon. Not. R. Astron. Soc., 168, 603-637 (1974)

[8] Kessel, O., Yorke, H.W. \& Richling, S. Photoevaporation of protostellar disks. III. The appearance of photoevaporating disks around young intermediate mass stars. Astron. Astrophys., 337, 832-846 (1998) 
[9] Hartmann, L., Kenyon, S.J. \& Calvet, N. The excess infrared emission of Herbig Ae/Be stars - Disks or envelopes? Astrophys J., 407, 219-231 (1993)

[10] Böhm, T. \& Catala, C. Forbidden lines in Herbig Ae/Be stars: the [O I](1F) 6300.31 and $6363.79 \AA$ lines. I. Observations and qualitative analysis. Astron. Astrophys., 290, 167-175 (1994)

[11] Miroshnichenko, A., Ivezic, Z. \& Elitzur, M. On Protostellar Disks in Herbig Ae/Be Stars. Astrophys J., 475, L41-L44 (1997)

[12] Pezzuto, S., Strafella, F. \& Lorenzetti, D. On the Circumstellar Matter Distribution around Herbig Ae/Be Stars. Astrophys J., 485, 290-307 (1997)

[13] Miroshnichenko, A., Ivezic, Z., Vinkovic, D. \& Elitzur, M. Dust Emission from Herbig AE/BE Stars: Evidence for Disks and Envelopes Astrophys J., 520, L115-L118 (1999)

[14] Barsony, M., Scoville, N.Z., Schombert, J.M. \& Claussen, M.J. The circumstellar environment of the emission-line star LkH-alpha 101. Astrophys J., 362, 674-690 (1990)

[15] Hou, J., Jiang, D. \& Fu, C. Physical properties of stellar winds from young stellar objects. Astron. Astrophys., 327, 725-735 (1997)

[16] Tuthill, P.G., Monnier, J.D., Danchi, W.C., Wishnow E.H. \& Haniff C.A. Michelson Interferometry with the Keck I Telescope. Publ. Astron. Soc. Pacif., 112, 555-565, (2000)

[17] Barsony, M., Schombert, J.M. \& Kis-Halas, K. The LkH-alpha 101 infrared cluster. Astrophys J., 379, 221-231 (1991)

[18] Herbig, G.H. The Spectrum of $\mathrm{LkH} \alpha 101$ in the Near-Infrared. Astrophys J., 169, 537-541 (1971)

[19] Stine, P.C. \& O'Neal, D. Radio Emission from Young Stellar Objects near LkH $\alpha 101$. Astron. J., 116, 890-894 (1998)

[20] Harris, S. $5 \mathrm{GHz}$ radio observations of $\mathrm{LkH} \alpha 101, \mathrm{M} 1-82 \sharp 1$ and other infrared sources. Mon. Not. R. Astron. Soc., 174, 601-607 (1976)

[21] Cohen, M., Bieging, J.H. \& Schwartz, P.R. VLA observations of mass loss from T Tauri stars Astrophys J., 253, 707-715 (1982)

[22] Panagia, N. Some Physical parameters of early-type stars. Astron. J., 78, 929-934 (1973)

[23] Millan-Gabet, R. et al. Sub-Astronomical Unit Structure of the Near-Infrared Emission from AB Aurigae. Astrophys J., 513, L131-L134 (1999)

[24] Millan-Gabet, R., Schloerb, F.P. \& Traub, W.A. Spatially resolved circumstellar structure of Herbig Ae/Be Stars in the Near-Infrared Astrophys J., 546, 358-381 (2001) 
[25] Akeson, R.L., Ciardi, D.R., van Belle, G.T., Creech-Eakman, M.J., \& Lada, E.A. Infrared Interferometric Observations of Young Stellar Objects Astrophys J., 543, 313$317(2000)$

[26] Gull, S.F. \& Skilling, J. Maximum entropy method in image processing. IEE Proc. Part F, 131, 646-650 (1984)

[27] Sivia, D.S. Phase Extension Methods. Thesis, Cambridge University (1987).

[28] Tuthill, P.G., Monnier, J.D. \& Danchi, W.C. A dusty pinwheel nebula around the massive star WR 104 Nature, 398, 487, (1999)

[29] Tuthill, P.G., Monnier, J.D., Danchi, W.C. \& Lopez B. Smoke Signals From IRC +10216: 1. Milliarcsecond Proper Motions of the Dust Astrophys. J., 543, 284, (2000)

Acknowledgements We would like to thank D. Sivia for the maximum-entropy mapping program "VLBMEM". Data were obtained at the W.M. Keck Observatory, made possible by the generous support of the W.M. Keck Foundation, operated as a scientific partnership among the California Institute of Technology, the University of California and NASA. This work was supported through grants from the National Science Foundation.

Correspondence and requests for materials should be addressed to Peter Tuthill (e-mail: gekko@physics.usyd.edu.au) 